\title{
Management of Sucking Pest of Tomato
}

\author{
V.J. Deshmukh, S.A. Patil and T.S. Kadgaonkar* \\ Biranje Panand, E word, Kasaba Bawada, Kolhapur, Maharashtra-416006, India \\ *Corresponding author
}

\begin{abstract}
Keywords
Tomato, White fly,

Thrips,

Management

Article Info

Accepted:

18 August 2018

Available Online:

10 September 2018 major pest viz. Whitefly (Bemisia tabaci) and Thrips (Thrips tabaci) of tomato during Rabi-2016.The modules consisted of M-1 Erection of yellow sticky traps + foliar spray of imidacloprid + spray of cypermethrin + spray fenazaquin at the appearance of mites; M-2 Erection of yellow sticky traps + spray of imidacloprid + spray of azadirachtin + spray fenazaquin at the appearance of mites; M-3 Erection of yellow sticky traps + spray of dimethoate + spray of indoxacarb + spray fenazaquinat the appearance of mites; M-4 Erection of yellow sticky traps + foliar spray of dimethoate+ spray of lambda-cyhalothrin + spray fenazaquin at the appearance of mites; M-5 Erection of yellow sticky traps+ foliar spray of imidacloprid + spray of chlorantraniliprole + spray fenazaquinat the appearance of mites and untreated check (M-6). The results revealed that minimum white flies attack on the module M1 (4.11whitefly/leaf) and next promising modules was M5 (4.18 whitefly/leaf) while in case of thrips minimum attack noticed in the module M1 (2.47 thrips/leaf) and next promising modules was M5 (2.60 thrips/leaf).
\end{abstract}

\section{A B S T R A C T}

Field experiment was conducted at farmer's field A/P Alate, Tal- Hatkangale, DistKolhapur to evaluate the effectiveness of different pest management modules against

\section{Introduction}

Tomato (Solanum lycopersicon L.) is one of the most popular and widely grown solanaceous crop in many countries including India. The estimated area under tomato in India is about 80.85 lakh ha with a production of 19.69 lakh tonnes and productivity 24.4 MT/ha of fruits (Anonymous, 2016-17). The tomato agro-ecosystem is characterized by having few major key pests and some minor or secondary pests. Tomato growers in Maharashtra regularly experienced the economic damage caused tomato fruit borer (Helicoverpa armigera Hubner), leaf miner (Liriomyza trifolii Blanchard), thrips (Thrips tabaci L.), white flies (Bemisia tabaci Gennadius), cutworm (Agrotis ipsilon Root), leaf eating caterpillar (Spodoptera litura Fabr.), aphid (Aphis gossypii Glov.), mealy bug (Ferrisia virgata Kill) and mites (Tetranychus sp.). Among the insect pests; sucking pests viz. thrips, whitefly and aphid do cause severe damage to crop by transmitting virus diseases rather than direct feeding. Therefore, the sucking pests should be considered as economically important.

\section{Materials and Methods}

Field experiment was conducted at Alate, TalHatkangale, Dist-Kolhapur, India situated at 
$616.27 \mathrm{~m}$ above mean sea level on $16^{0} 46^{\prime} 8.98^{\prime \prime} \quad$ North to $74^{0} 23$ '38.16'"East latitude on tomato variety, JK-811 in Randomized Block Design (RBD) during Rabi season 2016 with three replications. The plot size was $3.00 \times 2.25 \mathrm{~m}^{2}$ with $45 \times 60 \mathrm{~cm}^{2}$ spacing. Six management modules were taken including an untreated check with three replications. Details of modules were given in Table 1 . The seedlings were transplanted on $2^{\text {nd }}$ December 2016.

Observations were recorded one day before spray and after $30,40,55$, and 70 days after planting on all pests infesting tomato. Observations on pest count will be recorded on five randomly selected plants in each treatment plot on each selected plant, three leaves each from upper, middle and bottom portion were observed from lower side for presence of thrips and whiteflies.

Pre count will be taken one day prior to first spray. Collected data were then subjected to pooled analysis of variance (ANOVA) after appropriate transformations according to Panse and Sukhatme, (1967).

\section{Results and Discussion}

Data pertained in Table 2 and 3 on white fly and thrips infestation revealed that all the treatments were effective against the white fly and thrips, though varied in their efficacies for white fly (4.11-13.29) and for thrips (2.477.52) and were significantly superior to the check.

For white fly present observation showed that module M1 (4.11 whitefly/leaf) was found as the best module. The next promising modules was M5 (4.18 whitefly/leaf) and M3 (6.50 whitefly/leaf) and M2 (6.51 whitefly/leaf) were found equally effective in next order of efficacy. The module M1 and M5 recorded highest per cent reduction of whitefly over control i.e. $69.07 \%$ \& $68.55 \%$, respectively. Module M3 (51.09\%) and M2 (51.02\%) recorded similar result in per cent reduction of whitefly over control. Module M4 (46.20\%) was relatively less effective in controlling the whitefly. The present study is substantially supported by the findings of Dhar and Bhattacharya (2015), Kar (2017) and Kumar et al., (2017), Sarangdevot et al., (2006).

Table.1 Module details

\begin{tabular}{|c|c|}
\hline Mo. No. & MODULE DETAILS \\
\hline $\mathbf{M}_{1}$ & $\begin{array}{l}\left.\text { Erection of yellow sticky traps(1-2 traps @ } 50-100 \mathrm{~m}^{2}\right)+ \text { foliar spray of imidacloprid } 200 \mathrm{SL} @ 0.5 \mathrm{ml} / \text { lit } \\
\text { at } 20 \text { \& } 30 \text { DAT + spray of cypermethrin } 25 \mathrm{EC} @ 0.5 \mathrm{ml} / \text { lit at } 15 \text { days interval at the initiation of } \\
\text { flowering + spray fenazaquin } 10 \text { EC @ } 2 \mathrm{ml} / \text { lit at the appearance of mites. }\end{array}$ \\
\hline$\overline{\mathbf{M}_{2}}$ & $\begin{array}{l}\text { Erection of yellow sticky traps }\left(1-2 \text { traps @ } 50-100 \mathrm{~m}^{2}\right)+\text { foliar spray of imidacloprid } 200 \mathrm{SL} @ 0.5 \mathrm{ml} / \mathrm{lit} \\
\text { at } 20 \text { \&30 DAT + spray of azadirachtin @ } 2.5 \mathrm{ml} / \text { lit at } 15 \text { days interval at the initiation of flowering + } \\
\text { spray fenazaquin } 10 \text { EC @ } 2 \mathrm{ml} / \text { lit at the appearance of mites. }\end{array}$ \\
\hline$\overline{M_{3}}$ & $\begin{array}{l}\text { Erection of yellow sticky traps }\left(1-2 \text { traps @ } 50-100 \mathrm{~m}^{2}\right)+\text { foliar spray of dimethoate } 30 \mathrm{EC} @ 2 \mathrm{ml} / \text { lit at } 20 \\
\& 30 \mathrm{DAT}+\text { spray of indoxacarb } 14.5 \mathrm{SC} 1 \mathrm{ml} / \mathrm{lit} \text { at } 15 \text { days interval at the initiation of flowering + spray } \\
\text { fenazaquin10 EC @ } 2 \mathrm{ml} / \text { at the appearance of mites. }\end{array}$ \\
\hline $\mathbf{M}_{4}$ & $\begin{array}{l}\text { Erection of yellow sticky traps }\left(1-2 \text { traps @ } 50-100 \mathrm{~m}^{2}\right)+\text { foliar spray of dimethoate } 30 \mathrm{EC} @ 2 \mathrm{ml} / \text { lit at } 20 \\
\& 30 \text { DAT + spray of lambda-cyhalothrin5 EC @ } 0.8 \mathrm{ml} / \text { lit at } 15 \text { days interval at the initiation of flowering } \\
\text { + spray fenazaquin } 10 \mathrm{EC} @ 2 \mathrm{ml} / \text { lit at the appearance of mites. }\end{array}$ \\
\hline$\overline{\mathbf{M}_{5}}$ & $\begin{array}{l}\text { Erection of yellow sticky traps }\left(1-2 \text { traps @ } 50-100 \mathrm{~m}^{2}\right)+\text { foliar spray of imidacloprid } 200 \mathrm{SL} @ 0.5 \mathrm{ml} / \mathrm{lit} \\
\text { at } 20 \text { \& } 30 \mathrm{DAT}+\text { spray of chlorantraniliprole } 18.5 \mathrm{SC} @ 0.5 \mathrm{ml} / \mathrm{lit} \text { at } 15 \text { days interval at the initiation of } \\
\text { flowering + spray fenazaquin } 10 \text { EC @ } 2 \mathrm{ml} / \text { lit at the appearance of mites. }\end{array}$ \\
\hline $\mathrm{M}_{6}$ & Untreated control. \\
\hline
\end{tabular}


Table.2 Effect of different modules on whitefly (Bemisia tabaci)

\begin{tabular}{|c|c|c|c|c|c|c|c|}
\hline \multirow[t]{2}{*}{ Module } & \multicolumn{5}{|c|}{ Mean survival population of whitefly /leaf } & \multirow[t]{2}{*}{ Mean } & \multirow{2}{*}{$\begin{array}{l}\% \text { reduction } \\
\text { over control }\end{array}$} \\
\hline & Precount & 30 DAT & 40 DAT & 55 DAT & 70 DAT & & \\
\hline $\mathbf{M}_{1}$ & $\begin{array}{c}10.89 \\
(3.37)^{*}\end{array}$ & $\begin{array}{c}7.32 \\
(2.79)\end{array}$ & $\begin{array}{c}5.22 \\
(2.38)\end{array}$ & $\begin{array}{c}2.66 \\
(1.77)\end{array}$ & $\begin{array}{c}1.25 \\
(1.32)\end{array}$ & $\begin{array}{c}4.11 \\
(2.07)\end{array}$ & 69.07 \\
\hline $\mathbf{M}_{2}$ & $\begin{array}{r}10.82 \\
(3.36)\end{array}$ & $\begin{array}{c}7.38 \\
(2.81)\end{array}$ & $\begin{array}{c}5.15 \\
(2.37)\end{array}$ & $\begin{array}{c}6.20 \\
(2.59)\end{array}$ & $\begin{array}{c}7.31 \\
(2.79)\end{array}$ & $\begin{array}{c}6.51 \\
(2.64)\end{array}$ & 51.02 \\
\hline $\mathbf{M}$ & $\begin{array}{l}10.58 \\
(3.32)\end{array}$ & $\begin{array}{c}9.49 \\
(3.16)\end{array}$ & $\begin{array}{c}8.07 \\
(2.93)\end{array}$ & $\begin{array}{c}5.30 \\
(2.40)\end{array}$ & $\begin{array}{c}3.15 \\
(1.91)\end{array}$ & $\begin{array}{c}6.50 \\
(2.60)\end{array}$ & 51.09 \\
\hline$\overline{\mathbf{M}_{4}}$ & $\begin{array}{l}10.68 \\
(3.34)\end{array}$ & $\begin{array}{c}9.39 \\
(3.14)\end{array}$ & $\begin{array}{c}7.96 \\
(2.91)\end{array}$ & $\begin{array}{c}6.10 \\
(2.56)\end{array}$ & $\begin{array}{c}5.13 \\
(2.37)\end{array}$ & $\begin{array}{c}7.15 \\
(2.75)\end{array}$ & 46.20 \\
\hline $\mathbf{M}_{5}$ & $\begin{array}{l}10.87 \\
(3.37)\end{array}$ & $\begin{array}{c}7.21 \\
(2.77)\end{array}$ & $\begin{array}{c}5.02 \\
(2.35)\end{array}$ & $\begin{array}{c}2.95 \\
(1.85)\end{array}$ & $\begin{array}{c}1.54 \\
(1.42)\end{array}$ & $\begin{array}{c}4.18 \\
(2.10)\end{array}$ & 68.55 \\
\hline $\mathbf{M}_{6}$ & $\begin{array}{l}10.27 \\
(3.28)\end{array}$ & $\begin{array}{l}11.83 \\
(3.51)\end{array}$ & $\begin{array}{l}12.52 \\
(3.61)\end{array}$ & $\begin{array}{l}13.18 \\
(3.70)\end{array}$ & $\begin{array}{l}15.61 \\
(4.01)\end{array}$ & $\begin{array}{l}13.29 \\
(3.71)\end{array}$ & \\
\hline S. E. + & 0.114 & 0.084 & 0.081 & 0.088 & 0.090 & & \\
\hline C. D. at $\overline{5} \%$ & NS & 0.252 & 0.246 & 0.266 & 0.272 & & \\
\hline C. V. & & 5.52 & 5.91 & 7.11 & 7.85 & & \\
\hline
\end{tabular}

Table.3 Effect of different modules on thrips (Thrips tabaci)

\begin{tabular}{|c|c|c|c|c|c|c|c|}
\hline \multirow[t]{2}{*}{ Module } & \multicolumn{5}{|c|}{ Mean survival population of thrips /leaf } & \multirow[t]{2}{*}{ Mean } & \multirow{2}{*}{$\begin{array}{l}\% \text { reduction } \\
\text { over control }\end{array}$} \\
\hline & Precount & 30DAT & 40 DAT & 55 DAT & 70 DAT & & \\
\hline $\mathbf{M}_{1}$ & $\begin{array}{c}4.53 \\
(2.24)^{*}\end{array}$ & $\begin{array}{c}3.65 \\
(2.03)\end{array}$ & $\begin{array}{c}3.22 \\
(1.93)\end{array}$ & $\begin{array}{c}1.53 \\
(1.42)\end{array}$ & $\begin{array}{c}1.47 \\
(1.40)\end{array}$ & $\begin{array}{c}2.47 \\
(1.70)\end{array}$ & 67.15 \\
\hline$\overline{\mathbf{M}_{2}}$ & $\begin{array}{c}4.50 \\
(2.23)\end{array}$ & $\begin{array}{c}3.62 \\
(2.03)\end{array}$ & $\begin{array}{c}3.20 \\
(1.92)\end{array}$ & $\begin{array}{c}3.36 \\
(1.96)\end{array}$ & $\begin{array}{c}3.91 \\
(2.10)\end{array}$ & $\begin{array}{c}3.52 \\
(2.00)\end{array}$ & 53.19 \\
\hline $\mathbf{M}_{3}$ & $\begin{array}{c}4.35 \\
(2.20)\end{array}$ & $\begin{array}{c}4.08 \\
(2.13)\end{array}$ & $\begin{array}{c}3.81 \\
(2.07)\end{array}$ & $\begin{array}{c}2.50 \\
(1.72)\end{array}$ & $\begin{array}{c}2.37 \\
(1.69)\end{array}$ & $\begin{array}{c}3.19 \\
(1.90)\end{array}$ & 57.58 \\
\hline$\overline{\mathbf{M}_{4}}$ & $\begin{array}{c}4.55 \\
(2.24)\end{array}$ & $\begin{array}{l}4.04 \\
(2.13)\end{array}$ & $\begin{array}{c}3.85 \\
(2.08)\end{array}$ & $\begin{array}{c}3.40 \\
(1.70)\end{array}$ & $\begin{array}{c}3.45 \\
(1.98)\end{array}$ & $\begin{array}{c}3.69 \\
(1.97)\end{array}$ & 50.93 \\
\hline $\mathbf{M}_{\mathbf{5}}$ & $\begin{array}{c}4.49 \\
(2.23)\end{array}$ & $\begin{array}{c}3.60 \\
(2.02)\end{array}$ & $\begin{array}{c}3.19 \\
(1.92)\end{array}$ & $\begin{array}{c}1.82 \\
(1.51)\end{array}$ & $\begin{array}{c}1.78 \\
(1.55)\end{array}$ & $\begin{array}{c}2.60 \\
(1.75)\end{array}$ & 65.43 \\
\hline$\overline{M_{6}}$ & $\begin{array}{c}4.46 \\
(2.21)\end{array}$ & $\begin{array}{c}5.35 \\
(2.42)\end{array}$ & $\begin{array}{c}5.66 \\
(2.47)\end{array}$ & $\begin{array}{c}8.71 \\
(3.03)\end{array}$ & $\begin{array}{l}10.34 \\
(3.29)\end{array}$ & $\begin{array}{c}7.52 \\
(2.80)\end{array}$ & \\
\hline S. E. \pm & 0.084 & 0.070 & 0.077 & 0.090 & 0.099 & & \\
\hline C.D. at $5 \%$ & NS & 0.212 & 0.232 & 0.272 & 0.300 & & \\
\hline C. V. & & 6.63 & 7.46 & 9.54 & 9.97 & & \\
\hline
\end{tabular}

DAT $=$ Days after transplanting

*Figures in parentheses are transformed values $\sqrt{\mathrm{X}+0.5}$

For thrips present observation showed that the module module M1 (2.47 thrips/leaf) was found as the best treatment. The next promising modules were M5 (2.60 thrips/leaf). The module M3 (3.19 thrips/leaf) and M2 (3.52 thrips/leaf) were found equally 
effective in next order of efficacy. The module M1 and M5 recorded highest per cent reduction of thrips over control i.e. $67.15 \%$ \& $65.43 \%$, respectively followed by M3 (57.58\%), M2 (53.19\%) and M4 (50.93\%). The present study is substantially supported by findings of Muhammad et al., (2004), Muhammad et al., (2008), Raghuvanshi (2014) and Khan et al., (2014).

\section{References}

Anonymous. 2016-17. Indian Horticulture Database.

Dhar, T. and Bhattacharya, S. 2015. Efficacy of imidacloprid and spinosad against pest complex of okra and tomato. Int. J. Bio-res. Env. Agril. Sci., Vol. 1(3): 126131

Kar, A. 2017. Bioefficacy evaluation of imidacloprid $17.8 \% \quad$ SL and thiamethoxam against whitefly on tomato and their effect on natural enemies. J. Ento. Zoology Stud. 5(3): 1064-1067

Khan, S. B., Velo, S., Riffat, S., Abdul, R. A., Muhammad, N., Muhammad, N. S.
2014. Screening of biopesticides against Insect Pests of Tomato. European Acad. Res. Vol. II, Issue 5/August 2014

Muhammad, A., Muhammad, R., Syed, A. S. and Faheem, A. 2004. Comparative efficacy of different insecticides against sucking pests of cotton. J. res. Sci., 15(1), 53-58

Muhammad, R. A., Muhammad, A, Safdar, A. A. and Muhammad, H. B. 2008. Comparative efficacy of insecticides against sucking insect pests of cotton. Pak. J. Life Soc. Sci., 6(2): 140-142

Raghuvanshi, S., Bhadauria, N. S. and Pradyumn Singh, 2014. Efficacy of insecticides against major insect pests of soybean [Glycine max Merrill]. Trends in Biosciences, 7(3): 191-193.

Sarangdevot, S. S., Kumar, Ashok and Chundawat, G. S., 2006. Studies on bioefficacy of some newer insecticides against Bemisia tabaci and Amrasca biguttula biguttula on tomato in southern Rajasthan. Pestology, 30(5): $39-42$.

\section{How to cite this article:}

Deshmukh, V.J., S.A. Patil and Kadgaonkar, T.S. 2018. Management of Sucking Pest of Tomato. Int.J.Curr.Microbiol.App.Sci. 7(09): 2732-2735.

doi: https://doi.org/10.20546/ijcmas.2018.709.339 